\title{
New Capabilities and Discovered Interconnectivities for a Curriculum-Integrated Multicourse Model Rocketry Project
}

\author{
Matthew J. Traum ${ }^{1}$, Vincent C. Prantil, William C. Farrow, Hope L. Weiss \\ Mechanical Engineering Department, Milwaukee School Of Engineering, Milwaukee, WI
}

\begin{abstract}
To provide students a more coherent and cohesive view of the mechanical engineering curriculum, we created and are delivering a multicourse curriculum-integrated engineering project that permeates and unifies five required classes within our undergraduate curriculum: 1) Freshman Design, 2) Dynamics, 3) Numerical Analysis, 4) Fluid Mechanics, and 5) Thermodynamics. Students enrolled in these Rocket Project (RP) classes design, build, flight test, and analyze model rockets through hands-on exercises. These activities challenge students to work on different aspects of the same rocket project across all four years of their degree program.

Critical to the seamless collection and presentation of data and experimental/numerical techniques across five courses was the development of new laboratory, field, and simulation capabilities driven by our goal: to measure all unknown variables needed for rocket performance analysis and modeling in-house without reliance on external data. These needed capabilities included: 1) collecting acceleration and barometric altitude data from a model rocket flight, 2) simulating via computer rocket trajectories for comparison to actual measured altitudes, 3) evaluating rocket performance by numerical methods to validate modeling assumptions, 4) determining rocket drag coefficient as a function of Reynolds number for velocities relevant to a launch, and 5) measuring rocket motor thrust as a function of time as well as the energy density of the fuel used. As these capabilities were developed, additional course interconnectivities and opportunities for data sharing were discovered and exploited to further enrich the course experience for students.
\end{abstract}

\section{Introduction}

We are delivering to undergraduate mechanical engineering (ME) students at the Milwaukee School of Engineering (MSOE) multicourse curriculum-integrated aerospace engineering projects. The projects challenge students to work on different aspects of the same rocket project across all four years of their degree program. Students design, build, flight test, and analyze model rockets through hands-on projects that unify different ME classes by permeating five unique areas within our undergraduate curriculum: 1) Freshman Design, 2) Dynamics, 3) Numerical Analysis, 4) Fluid Mechanics, and 5) Thermodynamics. These classes will hereafter be referred to as Rocket Project (RP) classes. The educational benefits of this program for student participants are described elsewhere [1].

\footnotetext{
${ }^{1}$ The lead author gratefully acknowledges the Wisconsin Space Grant Consortium 2012-2013 Higher Education Incentives Award Program for the financial support it provided to make this project possible.
} 
To deliver this new hands-on content for RP classes, we developed new experimental and computer simulation capabilities at MSOE, which are described in this paper. Critically, we found that the RP courses were highly interdependent. In other words, as shown in Figure 1, a successful outcome in one class typically depended on the availability of information resulting from the activities of one or more other RP classes. The co-investigators' common passion for aerospace engineering offered a unifying thread and a natural collaborative opportunity that enabled needed data to be collected and presented seamlessly to students in five different required ME courses across all four program years.

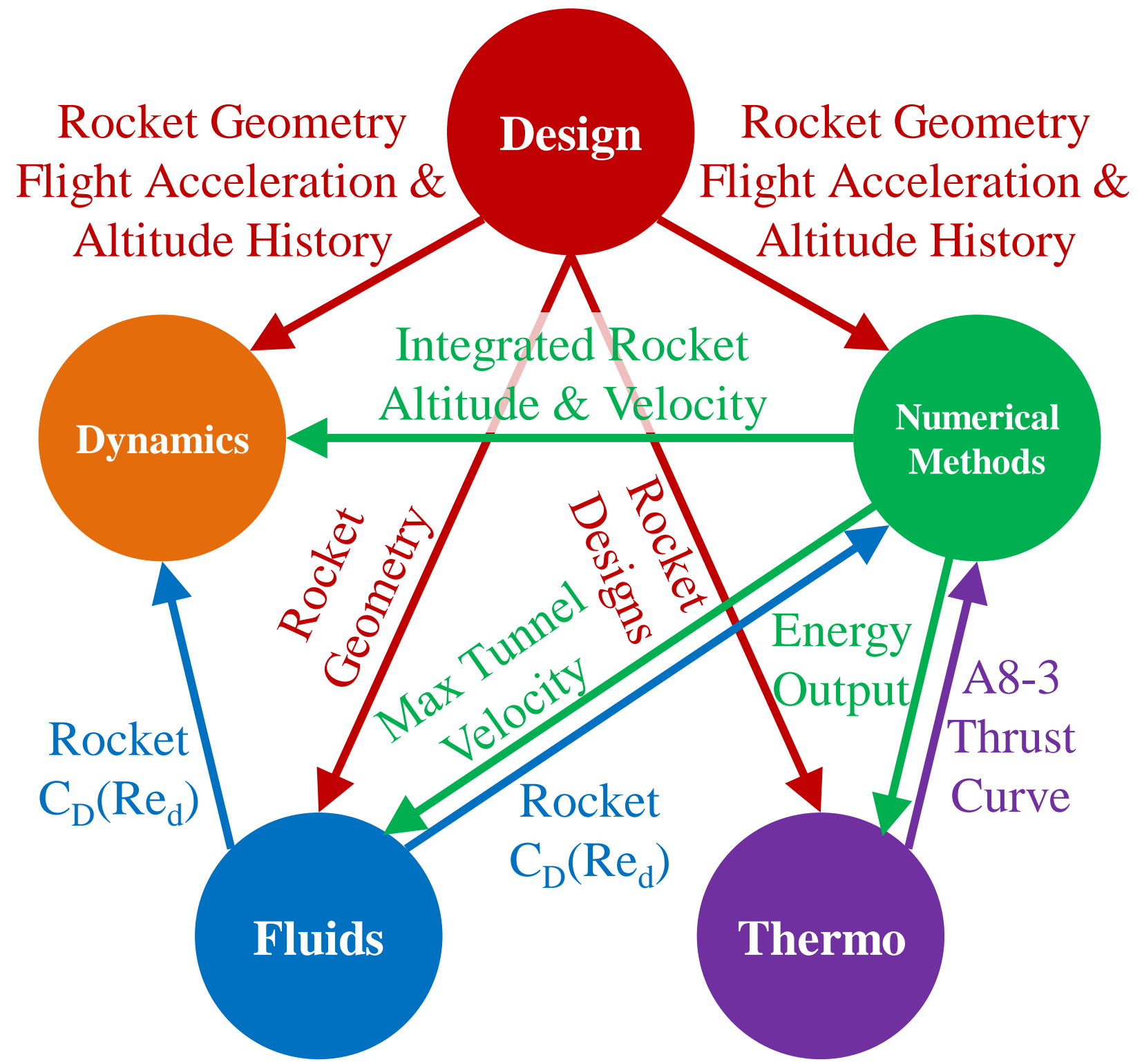

Figure 1: Interconnectivity map showing data, modeling, and analysis sharing across the five RP classes in this program. Since the classes were highly interdependent, successful outcomes in one class required availability of information resulting from one or more other RP classes. 


\section{METHODS}

We describe new capabilities developed in each RP class arising from cross-course data and information sharing. Courses are listed in the progression order they appear in the ME curriculum, which also corresponds to the most likely order in which students would encounter each sub-project: 1) Freshman Design, 2) Dynamics, 3) Numerical Analysis, 4) Fluid Mechanics, and 5) Thermodynamics.

\section{FRESHMAN DESIGN}

First in the sequence is the Freshman Design course, which develops students' basic solid modeling skills and teaches stages of the formal engineering design process. For their rocket project, students in this class were asked to satisfy the following identified need:

"A model rocket company needs a payload module that can carry a specific altimeter with embedded accelerometer on a model rocket in a way that will allow the altimeter to record the rocket's altitude as it flies skyward."

Constraints placed on the problem solution were that the design:

- Must not alter the altimeter/accelerometer in any way;

- Must be capable of sensing the altitude;

- Must allow the altimeter/accelerometer to be easily removed and reinstalled;

- Must allow the altimeter/accelerometer battery to be easily removed and replaced;

- Must allow for the activation of the altimeter/accelerometer, reset of the mode button. and confirmation of mode by viewing the mode LED;

- Must allow the flight data to be downloaded without having to remove the altimeter/accelerometer from the payload module;

- Must be sized so that all parts of the payload module fit within a $3.5 \times 3.5 \times 7$ inch volume (total rapid prototype volume available for each team); and

- Must be safe for a typical college student to operate.

After being assigned to project teams, students were supplied with a project kit that included the model rocket parts, the altimeter/accelerometer, and the basic tools needed to measure and assemble the rocket and payload. As part of their preparation for solving this design problem, teams had to reverse engineer the model rocket parts, digitize each part into a virtual solid model, and then assemble the parts into a virtual solid model of the entire rocket. Students' completed payload designs were fabricated using a solid laser sintering process in MSOE's Rapid Prototype Center (Figure 2). On the last weekend of the quarter in which the course is taught, each team launched their designs to collect acceleration and barometric altitude data from real flights; example data are shown in Figure 3.

At MSOE, the capabilities to 1) reverse engineer and digitize rocket solid models, 2) fabricate payload-carrying nosecones via rapid prototyping, and 3) to launch data-acquiring payloads on hobby-scale rockets all predate the multi-course rocket project. Previously, students in Freshman Design flew customized hobby-scale rockets with digital camera payloads in their nosecones that snapped downward-looking images at the vehicle's apogee [2].The new accelerometer/altimeter 
flight capability was the cornerstone of expanding rocketry use into many classes because the acceleration and altitude data collected were useful for numerical simulation, modeling, and validating analysis of other experiments (drag and trust measurement). The importance of this capability and the Freshman Design class is clear in Figure 1, which shows how information from this class is fed into the other four classes within the program.

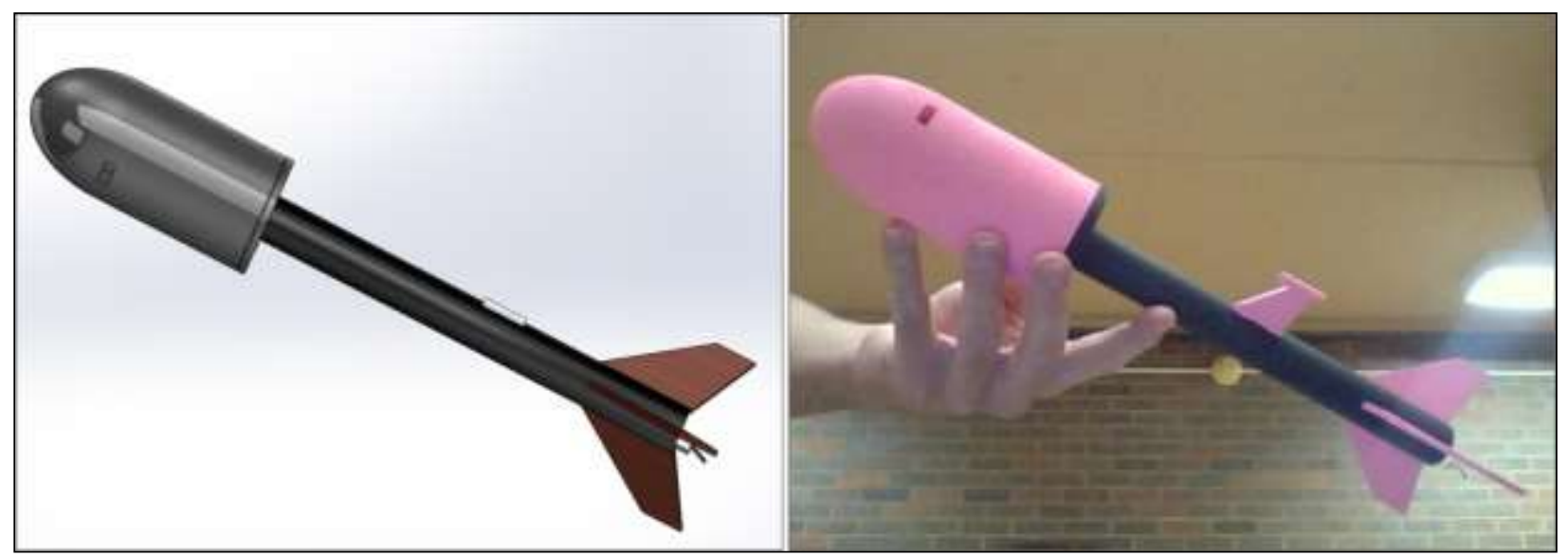

Figure 2: A mid-range sample of rocket payload module design to accommodate an altimeter. Designs in both virtual solid model (left) and physical model (right) forms are presented.

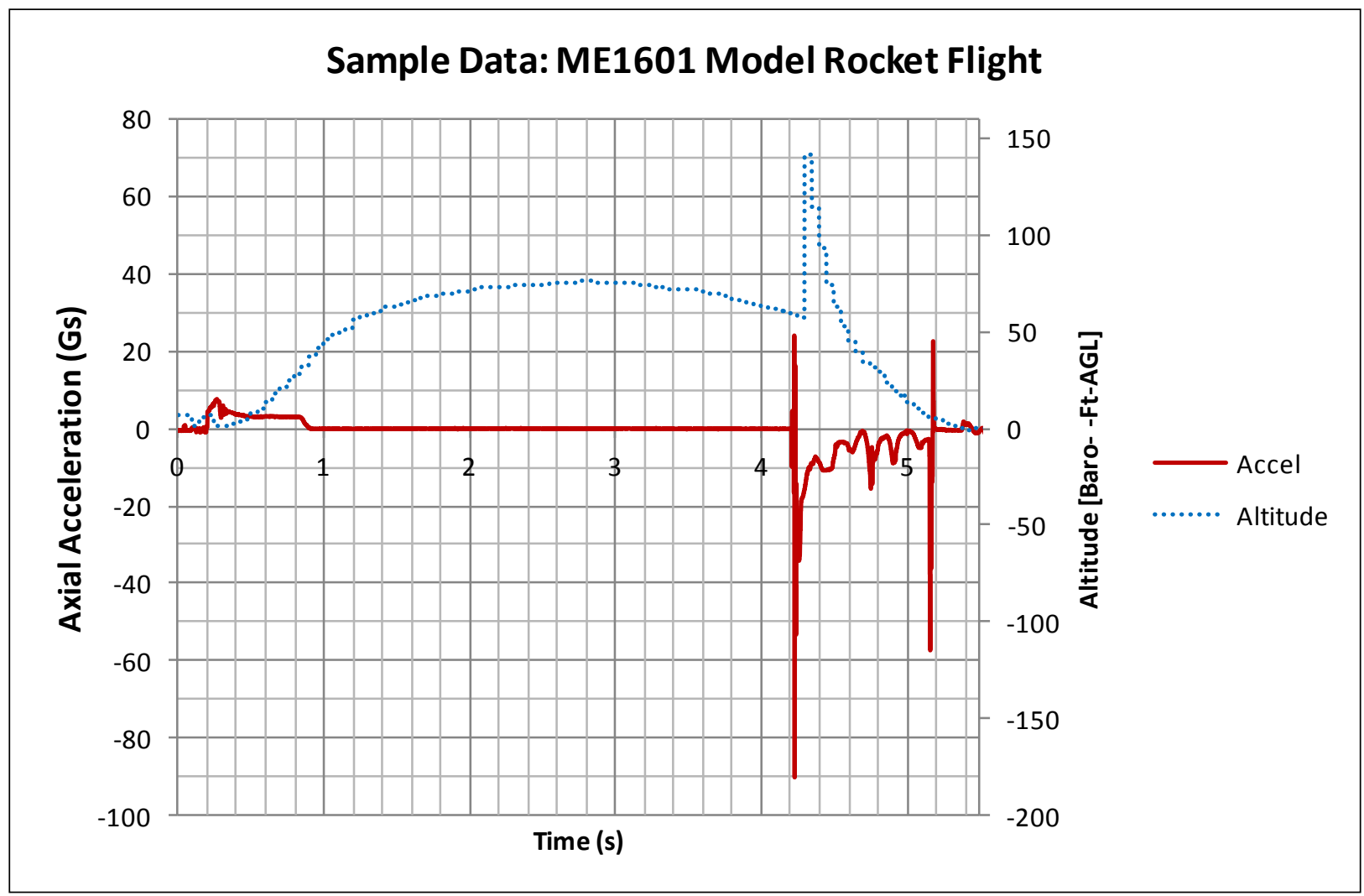

Figure 3: Sample flight data from a successful Freshman Design rocket launch with superimposed rocket altitude and acceleration. 


\section{DYNAMICS}

The second class in the multi-course RP sequence is sophomore-level Dynamics. This course incorporates computer laboratory sessions that apply use of numerical simulation and approximation in dealing with particle kinematics and kinetics concepts. With respect to the rocket project, students revisit their long-held modeling assumption from lower-division physics that air resistance can be neglected in particle trajectory calculations. Numerical modeling is performed that requires calculation of work due to non-conservative forces, primarily friction, and inclusion of velocity-dependent acceleration relations in curvilinear coordinate systems inherent in rocket flights from oblique launches. An idealized version of the drag model is emphasized with a frictional component added to the rocket acceleration wherein:

$$
a_{D R A G}=\frac{1}{2} \rho A C_{D} v^{2}\left(-\hat{e}_{t}\right)
$$

where $\rho$ is air density, $A$ is maximum rocket cross sectional area, $C_{D}$ is the drag coefficient, and $v$ is rocket velocity. In this equation, the direction of frictional acceleration, $\hat{e}_{t}$, always directly opposes the velocity of the rocket. Trajectory modeling must account for both this frictional acceleration as well as the gravitational acceleration, which is assumed to remain constant and be directed toward the Earth at all times.

To tie the rocket performance modeling work in Dynamics back to the actual rockets built and launched in Freshman Design, the cross-sectional area of student-designed nosecones, $A$, and the measured mass of student-designed rockets (complete with altimeter/accelerometer and battery payload) are used for calculations in Dynamics. Rocket drag coefficient, $C_{D}$, which is measured via a wind tunnel experiment in Fluid Mechanics later in the multi-course RP sequence (described below) is also given.

In the past, these rocket trajectory simulations were performed independently from real data. However, the newly developed RP course capability for Dynamics is our ability to compare simulated rocket trajectories to actual measured altitude using the altimeter/accelerometer data collected from actual flights in Freshman Design. Experimental velocity and altitude time histories obtained by analyzing in-flight acceleration data are determined in the subsequent Numerical Analysis course. From these experimentally-measured flight data can be gleaned the critical output predictions useful for model/experiment comparison in Dynamics: maximum range, maximum altitude, and flight time.

\section{NUMERICAL ANALYSIS}

Third in the multi-course RP sequence is junior-level Numerical Analysis class. This class devotes substantial time to model construction: simplifying physical assumptions, validation of numerical simulation results, and model iteration. By revisiting physical assumptions inherent in the models developed earlier in Dynamics, the rocket trajectory is simulated using techniques that are much more sophisticated. The following modeling processes are carried out: 
1. Double numerical integration of in-flight acceleration data [obtained from the Freshman Design course] to obtain experimental velocity and altitude time histories [which are used for calculations in the Dynamics class] as well as flight trajectories for oblique launches;

2. Calculation of work performed by aerodynamic drag forces [which is used in the Thermodynamics course];

3. Direct comparison of model/flight simulation velocity, altitude, and trajectory with three velocity-dependent drag model assumptions;

4. Determination of model prediction sensitivity to:

a. Impulse delivered assuming a square pulse thrust profile, or

b. Impulse delivered using experimental test data from a typical A8-3 Estes rocket motor [obtained from the Thermodynamics course]; and

5. Determination of model prediction sensitivity to assumptions regarding rocket payload weight including:

a. Assuming constant rocket weight, or

b. Accounting for mass fuel burn

As a baseline, constant rocket mass and constant step input (square pulse) thrust are assumed for the rocket. Using Newton's second law, the governing differential equation is

$$
m \frac{d v}{d t}=T-W-F_{D}(v)
$$

Where $m$ is the constant mass of the rocket, $v$ is the velocity of the rocket, $T$ is a constant thrust delivered over a specified amount of time, $W$ is the weight of the rocket, and $F_{D}(v)$ is the drag force on the rocket given as a function of velocity. Three drag models are evaluated: 1) No drag $\left.F_{D}(v)=0 ; 2\right)$ Linear $\operatorname{drag} F_{D}(v)=b v$ where $b$ is a linear coefficient of drag [which is instructorprovided, but based on limited theory with no experimental data]; and 3) Aerodynamic drag $F_{D}(v)=1 / 2 \rho C_{D} A v^{2}$ where $\rho$ is the density of air, $C_{D}$ is the drag coefficient [measured by wind tunnel testing in the Fluid Mechanics course] and corroboration with literature-published values [3], and $A$ is the cross-sectional area of the rocket (as built in Freshman Design). The velocity of the rocket is updated employing the simplest explicit time integration of Eqn. 2

$$
v_{i+1}=v_{i}+\frac{1}{m}\left(T-W-F_{D}\left(v_{i}\right)\right)
$$

using Euler's forward method (here $i$ indicates the time step). Mass of the rocket as a function of time is estimated using experimental thrust data (collected in the Thermodynamics course) assuming a constant fuel burn rate [4]. The governing equation now becomes

$$
m(t) \frac{d v}{d t}=T(t)-W(t)-F_{D}(v)
$$

Again, Euler's forward method is used to update the velocity 


$$
v_{i+1}=v_{i}+\frac{1}{m_{i}}\left(T_{i}-W_{i}-F_{D}\left(v_{i}\right)\right) .
$$

The height achieved by the rocket is determined numerically using Euler's forward method. As before, the in-flight acceleration data are integrated using the trapezoidal rule once to obtain the velocity and twice to obtain the height.

Prior to being incorporated as a RP course, the above Numerical Analysis exercise was conducted using rocket acceleration data created by the instructor. We now have the capability to perform flight simulations by working from real experimental flight data. The experimental rocket velocity is plotted in Figure 5, along with the velocities found using the linear drag model and the aerodynamic drag model with constant mass and with variable mass. We also have the capability to compare simulated rocket altitude time histories with experimental altimeter data. Figure 6 shows the rocket height from the experimental flight compared to predicted model heights using constant and variable mass along with the linear drag and aerodynamic drag models.

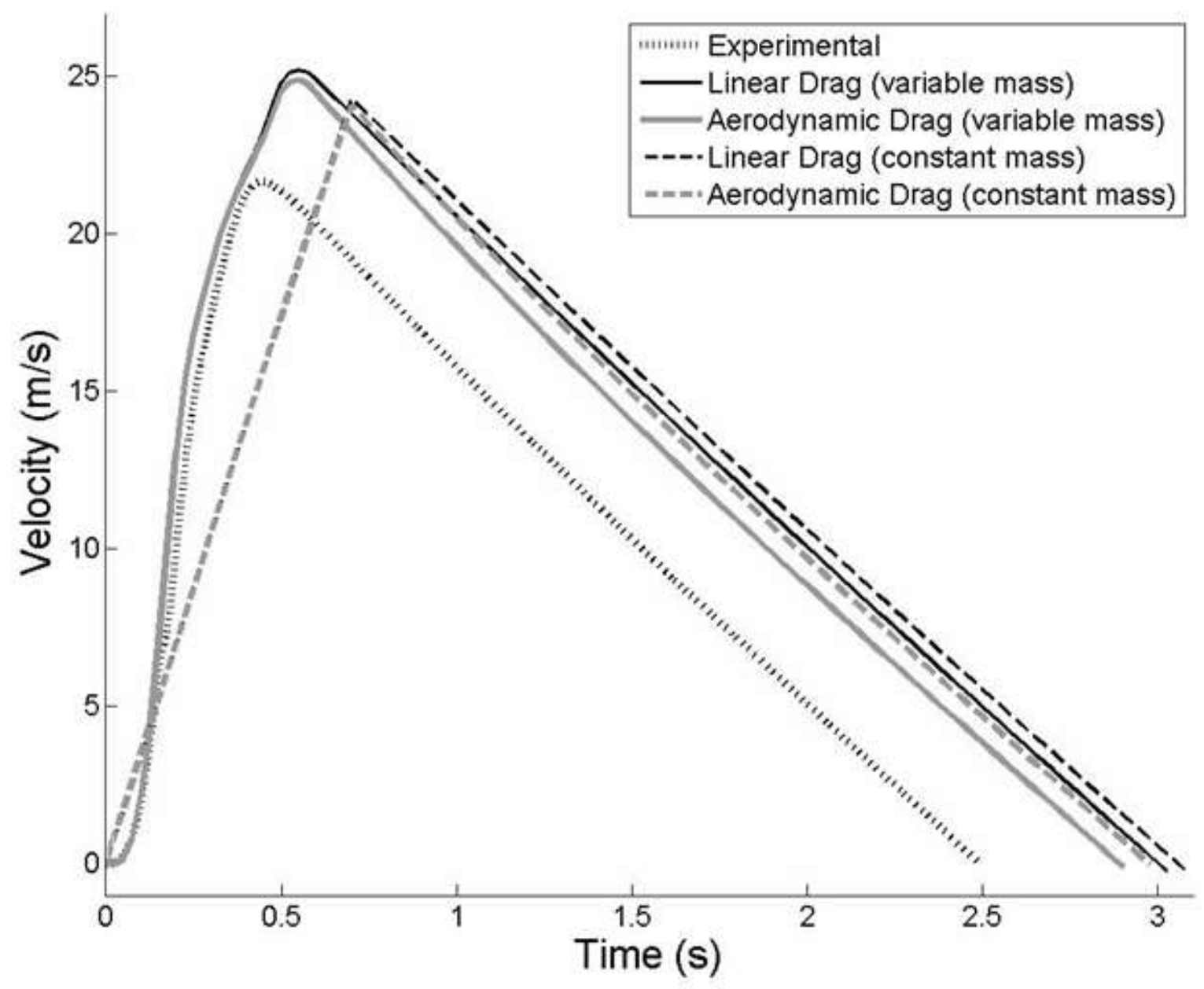

Figure 5: Rocket velocity as a function of time from the experimental flight compared against velocity history predicted by the model using constant and variable mass. The linear drag and aerodynamic drag models are shown. 


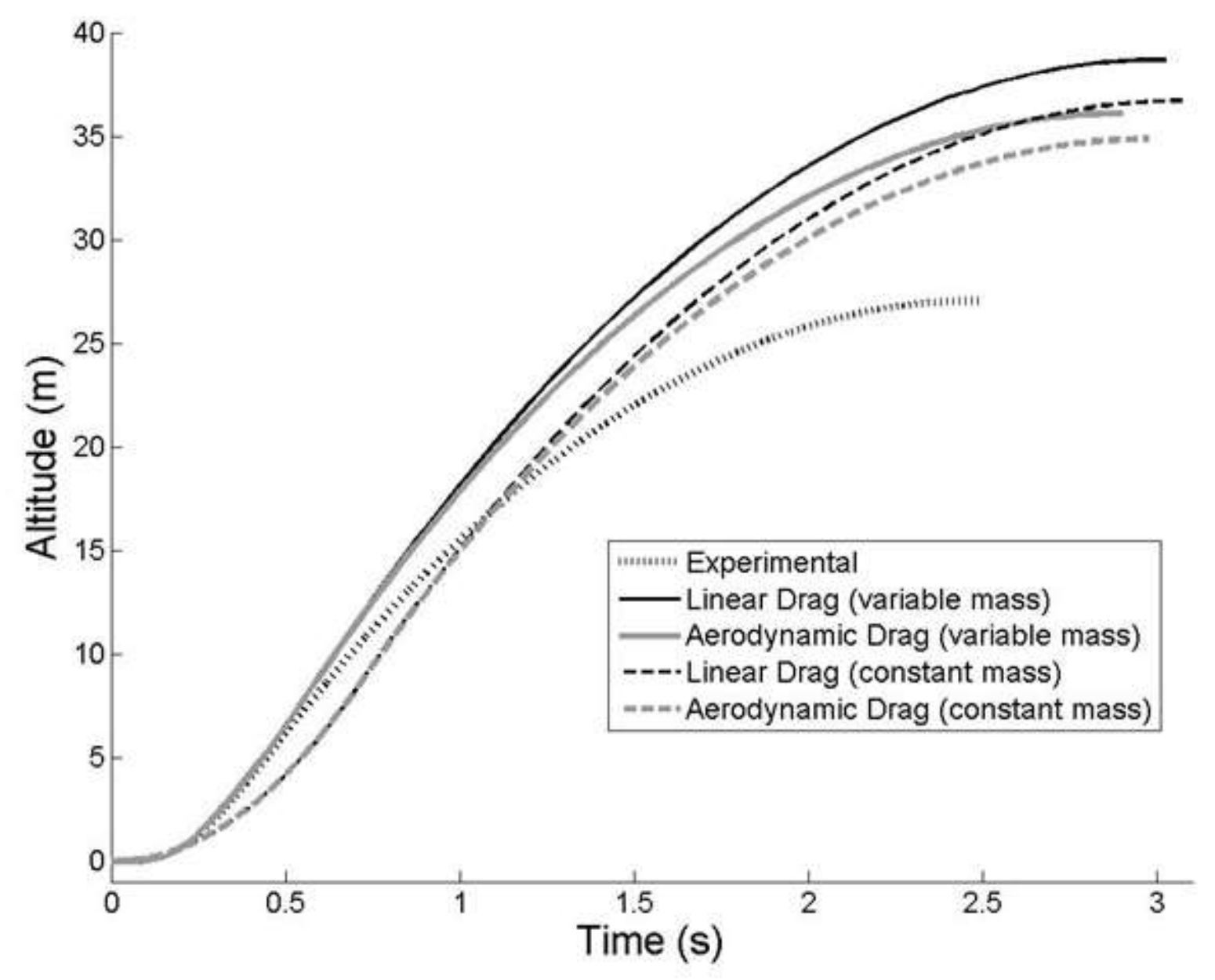

Figure 6: Rocket altitude from experimental flight data compared against altitude predicted from simulation using constant and variable mass. The linear drag and aerodynamic drag models are shown.

\section{FLUID MECHANICS}

The fourth class in the multi-course RP sequence is junior-level Fluid Mechanics for which a wind tunnel experiment was devised. Rocket drag was measured as a function of tunnel velocity, and the dimensionless variant (drag coefficient as a function of Reynolds Number) was also determined. The ability to make these measurements was a new capability developed for the rocket project to address the need to know rocket drag coefficients for the simulations performed in Dynamics and Modeling and Numerical Analysis.

As shown in Figure 7, the model rocket was initially hung in the tunnel's working section with the turbine off using two very thin threads positioned around the rocket's center of gravity. The rocket's mass was measured in advance of the experiment. A tripod-mounted digital camera captured the location of the rocket relative to a T-square, which indicated the reference vertical. A laser beam was directed at $45^{\circ}$ from horizontal to calibrate the image against unintended angle offsets between the camera and the working section. 
The wind tunnel was then turned on, and its velocity was measured using a pitot-static probe. Drag induced in the horizontal direction caused the rocket model to swing backward and assume a new equilibrium orientation. The new angle between the suspending rocket threads and horizontal was photographed for later measurement using digital image analysis software. The resulting drag coefficient was determined using Eqn. 6,

$$
D=\frac{m g}{\cos \Theta}
$$

where $m$ is the rocket mass, $\mathrm{g}$ is the gravitational acceleration, and $\theta$ is the angle between the suspension threads and horizontal. Reliable data were taken at tunnel speeds as high as $32.9 \mathrm{~m} / \mathrm{s}$, which is faster than $\sim 25 \mathrm{~m} / \mathrm{s}$ achieved by the rocket, as determined from experimental flight data (see Figure 5).

A plot of drag force versus tunnel velocity was produced from these measurements. The data where then nondimensionalized using rocket geometric and air fluid dynamic parameters to create a plot of drag coefficient versus Reynolds number, as shown in Figure 8. Apparent in Figure 8 is the drop in drag coefficient with increasing Reynolds number (i.e. tunnel velocity).

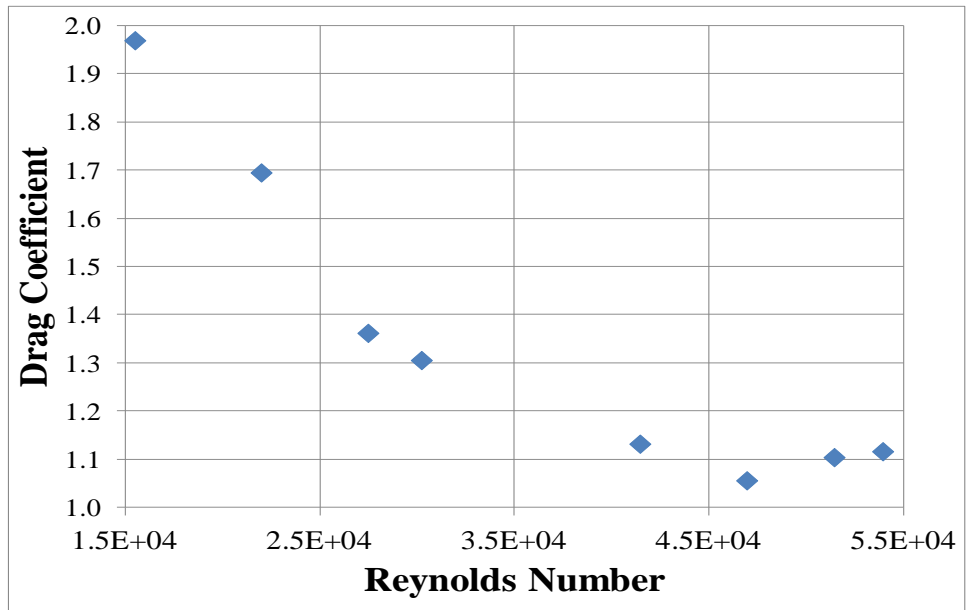

Figure 8: Rocket model drag coefficient as a function of Reynolds number measured in the Fluid Mechanics component of the rocket project. As with drag coefficient over a simple shape, like a sphere, drag coefficient is a strong function of Reynolds number for laminar flow, but this dependence disappears as flow transitions to turbulent.

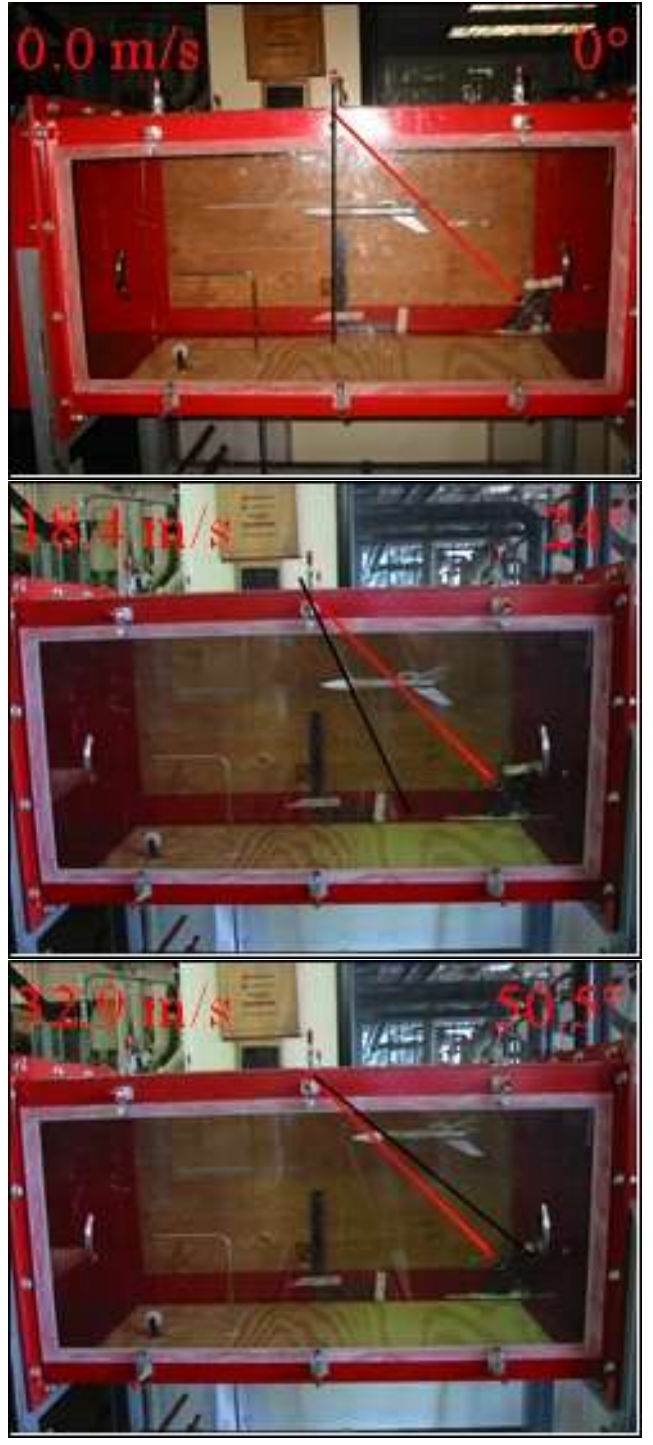

Figure 7: Model rocket drag coefficient as a function of velocity is experimentally measured in a wind tunnel. By suspending models about their center of mass by two threads. Wind tunnel velocity is increased and the resulting angle between the horizontal and the thread (black lines) is photographed for measurement. A laser beam projected $45^{\circ}$ from horizontal (red lines) corrects for any systematic error in angle between the tunnel working section and tripodmounted camera. 
Above Reynolds number of about 40,000 the drag coefficient seems to lose its functional dependence on Reynolds number. Qualitatively, this behavior is consistent with external flow over three-dimensional objects in that increasing Reynolds number generally decreases drag coefficient until the flow transitions to the fully turbulent regime where Reynolds number no longer has an effect on drag coefficient.

\section{THERMODYNAMICS}

The fifth multi-course RP class in the sequence is senior-level Thermodynamics. Here, an experimental thrust measurement apparatus was constructed that can accommodate model rocket motors in the $13.0 \mathrm{~mm}, 18.0 \mathrm{~mm}$, and $24.0 \mathrm{~mm}$ diameter size classes. Thus, any hobby-scale solid rocket motor from the 1/4A up to $\mathrm{E}$ total impulse classes can be tested. This capability was newly developed at MSOE for the multi-course rocket project to provide experimental motor thrust curves for the Numerical Analysis course.

The motor is securely mounted in a vertical orientation atop a compression load cell and ignited so exhaust is vented through a hood out of the test cell (Figure 9). A data acquisition system collects thrust data at 0.002 -second intervals and a low-passfilter, implemented in the data collection software, eliminates high frequency vibration artifacts to produce a smooth thrustversus-time curve.

To validate the thrust measurement apparatus, thrust curves were compared against curves published by the manufacturer based on data acquired through testing by the National Association of Rocketry (NAR), as shown in Figure 10. A useful

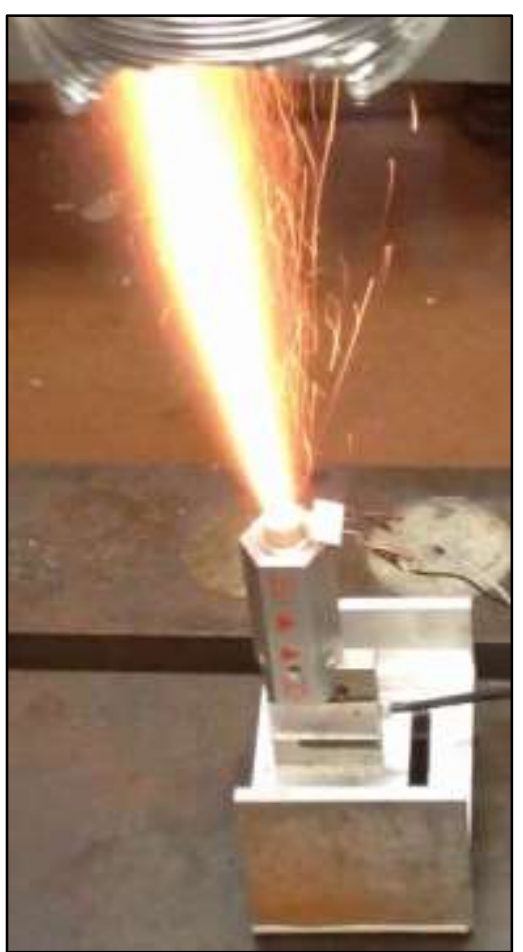

Figure 9: An Estes A8-3 model rocket engine is tested on the motor thrust apparatus. repository of hobby rocket motor thrust curve data is archived on-line at www.thrustcurve.org [5].

For the specific Estes A8-3 motor shown tested in Figure 9, the experimental thrust apparatus under-measures manufacturer-stated maximum thrust by $5.3 \%$, under-measures total impulse by $8.2 \%$, under-measures burn time by 0.152 seconds, and under-measurers average thrust by $2.7 \%$. However, uncertainty in experimental data was not quantified, and these data represent a single engine test. As pointed out by Haw [6], repeated tests generating enough data to enable statistical analysis would provide the most accurate global representation of motor performance.

In addition to obtaining motor thrust curves, experimental data were also used to estimate the energy density of black powder rocket motor fuel. The difference between pre-fire and post-fire motor masses gives an estimate of the mass of black powder consumed. So, if total motor output energy during launch is measured, the motor's energy density can be calculated. Most hobby motors include delay and recovery charges, which do not contribute to the measured output thrust but do contribute to the pre-fire/post-fire mass difference. To reduce the resulting error in 
determining rocket fuel energy density, booster motors without delay charges (A8-0 motors) were used for these measurements, leaving only the recovery charge as a small unaccounted mass in the overall energy balance.

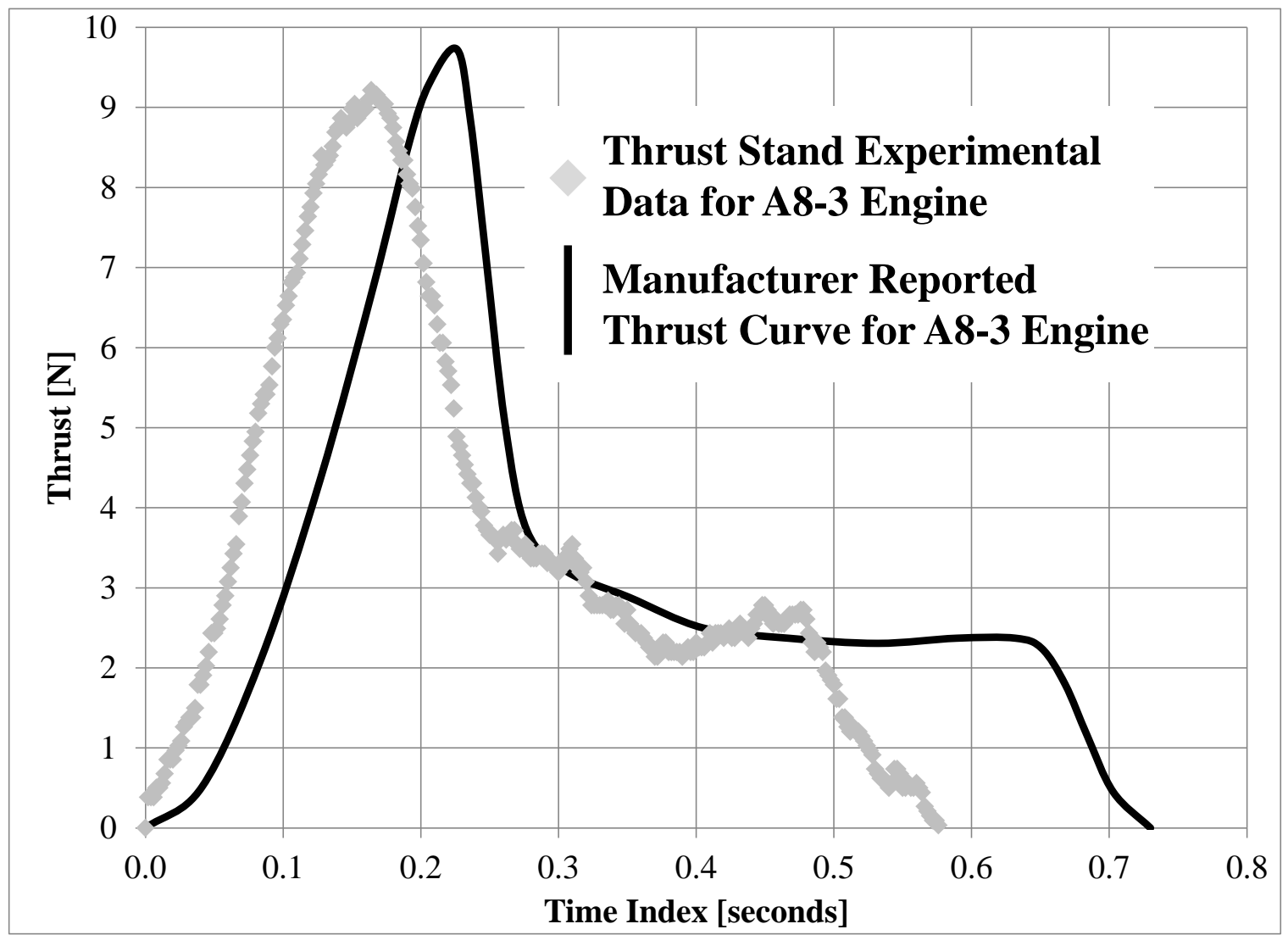

Figure 10: Comparison of manufacturer reported thrust curve for an Estes A8-3 model rocket engine versus experimental data collected using the motor thrust apparatus.

The resulting energy output is then validated in two ways. First, in the Numerical Analysis course, the energy output during a rocket launch is evaluated by combining dissipation from drag with the potential energy achieved at rocket apogee. This result directly benchmarks the product of motor energy density and mass consumed during static motor tests. Second Thermodynamics combustion analysis techniques are applied to black powder solid rocket motor fuel (charcoal and sulfur fuel combined with potassium nitrate oxidizer) to determine the specific energy released during the combustion process given formation enthalpies of the reactants and products:

$$
8 \mathrm{C}+3 \mathrm{~S}+10 \mathrm{KNO}_{3} \rightarrow 2 \mathrm{~K}_{2} \mathrm{CO}_{3}+3 \mathrm{~K}_{2} \mathrm{SO}_{4}+6 \mathrm{CO}_{2}+5 \mathrm{~N}_{2}
$$

Specific energy, e, obtained from this combustion analysis can then be compared against the energy released during experimental tests as represented by the thrust curve. 


$$
e=\frac{1 / 2\left(m_{f, i}-m_{f, f}\right)}{m_{f, i}} v_{e}^{2}
$$

where $m_{f, i}$ and $m_{f, f}$ are respectively initial and final fuel mass and $v_{e}$ is nozzle exit velocity. This velocity can be determined exactly via the following equation [7]:

$$
v_{e}=\frac{\int_{o}^{t} F(t) d t}{\int_{0}^{t} \dot{m}(t) d t}
$$

The numerator is calculated numerically as the area under the experimentally-measured thrustversus-time curve. However, the function for instantaneous expulsion of mass, which appears in the denominator, cannot be determined from available experimental data as the rocket motor mass change cannot be separately measured from thrust data. Thus, the mass expulsion rate is assumed to be linear, $\dot{m}(t) \approx\left(m_{f, i}-m_{f, f}\right) / \Delta t$, which is a reasonable engineering assumption for solid rocket motor grain that presents a constant area combustion front.

\section{CONCLUSIONS}

The authors collaborated across conventional ME Department division boundaries to create and deliver a multicourse curriculum-integrated rocket project that permeates and unifies five different classes within the ME undergraduate curriculum: 1) Freshman Design, 2) Dynamics, 3) Numerical Analysis, 4) Fluid Mechanics, and 5) Thermodynamics.

Seamless collection and presentation of data and experimental/numerical techniques across five courses was facilitated by the creation of new laboratory, field, and simulation capabilities at MSOE driven by our goal: to measure all unknown variables needed for rocket performance analysis and modeling in-house without reliance on external data. These needed capabilities included: 1) collecting acceleration and barometric altitude data from a model rocket flight, 2) simulating via computer rocket trajectories for comparison to actual measured altitudes, 3) evaluating rocket performance by numerical methods to validate modeling assumptions, 4) determining rocket drag coefficient as a function of Reynolds number for velocities relevant to a launch, and 5) measuring rocket motor thrust as a function of time as well as the energy density of the fuel used. As these capabilities were developed, additional course interconnectivities and opportunities for data sharing were discovered and exploited to further enrich the course experience for students.

\section{BIBLIOGRAPHY}

[1] M. J. Traum, V. Prantil, W. Farrow, H. Weis, "Interconnecting the Mechanical Engineering Curriculum Through An Integrated Multicourse Model Rocketry Project," Proceedings of the 120th American Society for Engineering Education (ASEE) Conference and Exposition, Atlanta, GA, June 23-26, 2013.

[2] W. C. Farrow, "Freshman Engineering Video-Rocket Design Project," Proceedings of the $19^{\text {th }}$ Annual Wisconsin Space Conference, Milwaukee, WI, August 13-14, 2009. 
[3] S. Niskanen, "Development of an Open Source Model Rocket Simulation Software", M.S. Thesis, Engineering Physics, Information and Natural Sciences, Helsinki University, May 20, 2009.

[4] K. Penn, W. V. Slaton, "Measuring Model Rocket Engine Thrust Curves," The Physics Teacher, Vol. 48, Issue 9, 2010, pp. 591.

[5] J. Coker, “Thrustcurve.org - Rocket Motor Performance Data On-Line," Web Site URL: http://www.thrustcurve.org/ (last accessed 4/6/3013).

[6] M. Haw, "A8-3 Model Rocket Impulse Measurement," The University of British Colombia cIRcle open access digital repository, Web Site URL: http://hdl.handle.net/2429/7905.

[7] G. P. Sutton, O. Biblarz, Rocket Propulsion Elements, $8^{\text {th }}$ Edition, Chapter 2, John Wily \& Sons, Hoboken, NJ, 2010, pp. 26-45. 